Health \& Medicine | Marco Balenci

\section{A holistic approach to cancer}

The unseen influence of the unconscious mind

Although the mind-body
dualism has dominated
Western medicine for
centuries, contemporary
cancer research is making
room for more holistic
approaches to understanding
carcinogenesis. Dr Marco
Balenci, psychoanalyst and
member of the American
Psychological Association,
looks at cancer as a systemic
rather than local disease.
His recent article places the
work of Elida Evans in the
history of American medical-
psychological thought and,
at the same time, presents
an updated picture of cancer
research from a holistic
standpoint.

ncreasing understanding of the mind-body connection is starting to shift traditional approaches thealth and disease - that is, the mind-boy dualism which dominated

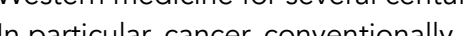
in particular, cancer, conventionally is being looked at through the lens of holistic medicine, dedicated to the whole patient - body and mind. It is a poorly known fact that Carl Gustav Jung's theory has influenced psychooncology, a field which examines the role of a cancer patient's emotions and experiences in the physiological mechanisms associated with cancer.

\section{JUNGIAN THEORY}

Jung, a Swiss psychiatrist, was one of the first psychoanalysts. His contribution to the understanding of medical and biological aspects of en than heis work in this abstantis even though his work in this area is practitioners. In fact, Jung, best know for a philosophical orientation of depth psychology, held a monistic view, providing seminal arguments for the ming-beminal argum Indeed, carcinogenesis (the formation of cancer) is currently ttributed to interactions between biological, psychological and sociological risk factors in a way that does not fit dualism.

Jung's theory has served as a foundation to the first work on cancer in the field of modern holistic medicine, A psylishological study of cancer, published in 1926 by Elida Evans. In it, Evans interpreted the psychology
of cancer patients in the light of Jung's
Psychological types, published in 1921 At the time, Evans, aware she was going against the stance commonly accepted "the medical profession, collected. "the emotiona history of the individual ", history of each patient.

Recognising that cancer is mainly a disease of the second part of life, Evans connected carcinogenesis to a highlighted the potentially destructive a genetically inherited entity, affecting human physiology and ideas. Carcinogenesis is described as a regressive energy process following a traumatic event of loss, after a life of relinquishing personal creativity to sustain relationships. Over time, this process resulted in withdrawal In 1953, even the American Psychosomatic Society was reluctant in by its president, George Engel. This resistance notwithstanding researchers interested in holistic medicine in Germany around the same time, spoke of "cancer psyche", a concept whereby cancer was a disease of the whole organism. This kind of theory laid the groundwork for the since-expanding field, aiming to in the development of disease.

PSYCHONEUROIMMUNOLOGY AND CANCER

The biopsychosocial model of aspects of the collective unconscious, and psychobiological collapse, which understand the mind-body interactio in the United States, acknowledging the narrowness of the biomedical model and announcing openness social, and environmental factors the examination of illness.

This framework saw the psychobiological unity of man and proposed that all diseases are conditioned by psychosocial factors including cancer. At the time, George Engel and his team could anticipate cancer in women who met the criteria for hopelessness-prone personality, whereby hopelessness was seen as the process that activated disease. Due to its non-specificity, this approach didn't take hold until 1975 when it reemerged as psychoneuroimmunology.

This discipline examines the interaction between the nervous, endocrine, and immune systems, providing evidence between the chemicals involved in these systems. For example, a large body of research has documented the presence of neuropeptides and their receptors throughout the body, suggesting a whole-body, physiologica basis of emotions. Moreover, studies from this discipline have shown that psychology and behaviour can impact functioning of the immune system, contributing to cancer incidence or progression through alterations of
immune cell activity or DNA repair.

PSYCHOANALYTICAL AND BEHAVIOURAL RESEARCH ON CANCER

From the 1950s, the notion of cancer as a "form of passive suicide" was supported by New York clinical psychologist Lawrence LeShan who argued that cancer patients had a "weak will to live," symptomatic of excess adaptation, whereby future cancer patients were more concerned with the opinions of others than the needs of their own self. Consequenty. they lived other-directed lives and presented a lack of individuation. Moreover, LeShan found that cancer patients had experienced the serious loss of a situation or a relationship. Hence, his ideas show that LeShan is a follower of Elida Evans, whose work most of her observations.

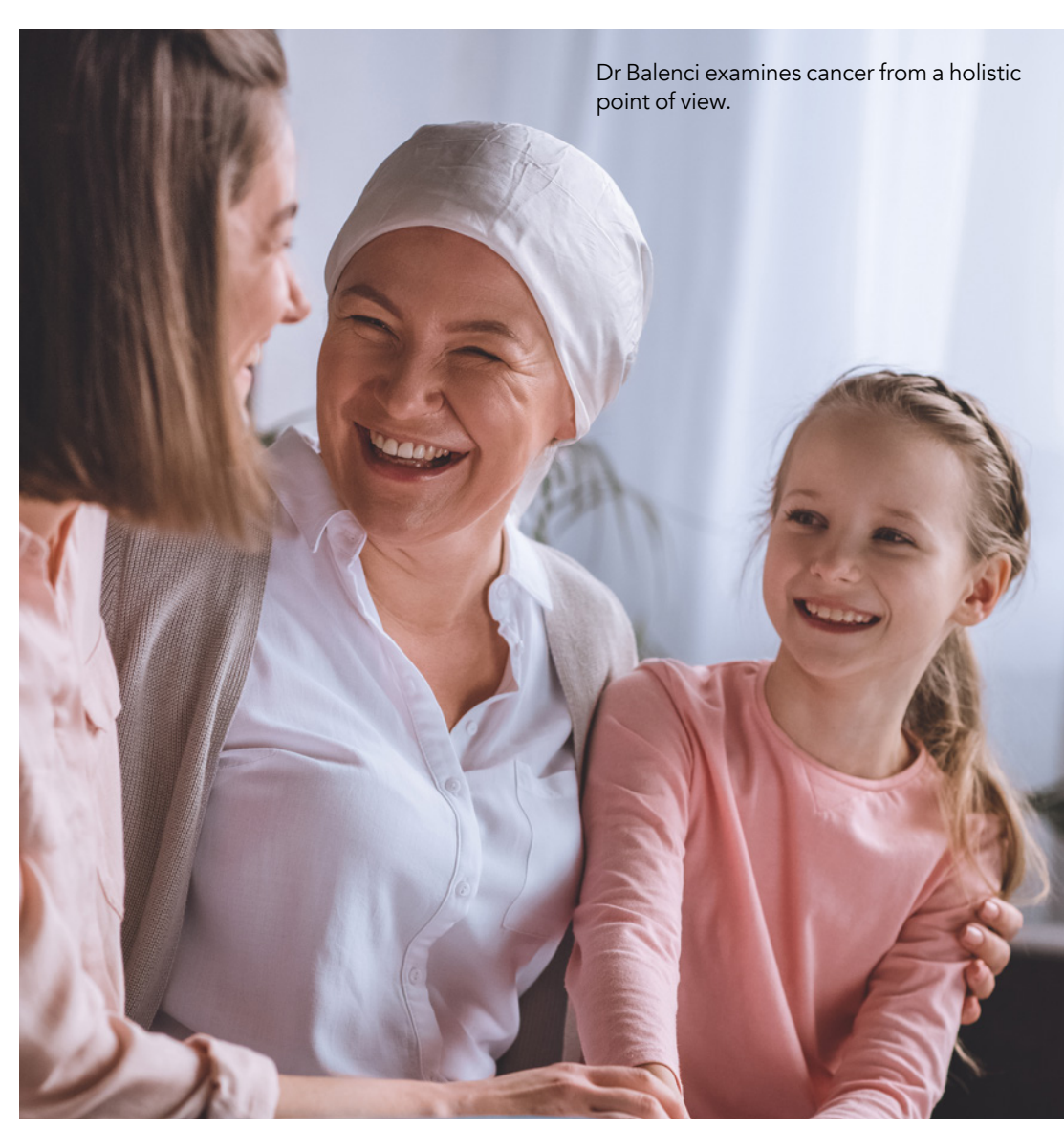

Although cancer has been seen as an entirely organic condition in modern times, recent research supports the hypothesis that cancer might be affected by emotional factors.

Shortly thereafter, oncology and psychology separated entirely, putting style of coping is associated with less and psychoanalysis, until the 1970 when behavioural medicine found th certain behavioural features might be more prone to cancer than others. Type C, characterised by behaviour. that is appeasing, cooperative, selfsacrificing, unassertive, and free of negative feelings has been identified as cancer prone. According to Steven Greer and Lydia Temoshok, however, this behaviour was not a determinant of cancer, but an important risk factor for it. This finding has received some supporting evidence in biophysiological research, showing that non-expression of emotions, which is the toxic core of Type $C$, is associated with a reduction faster cell multiplication at the and cancer patients.

\section{SOCIAL RISK FACTORS} FOR CANCER been introduced to modern cancer proposes it as a "symptom of losing existential encounter" between a person and the world, which has been industrialisstion. In this context, cancer is seen as going "together with mass society", raising criticism of contemporary lifestyles, particularly in the West. Psychologists have described contemporary functioning as a form of the original self to eliminate anxietyin 


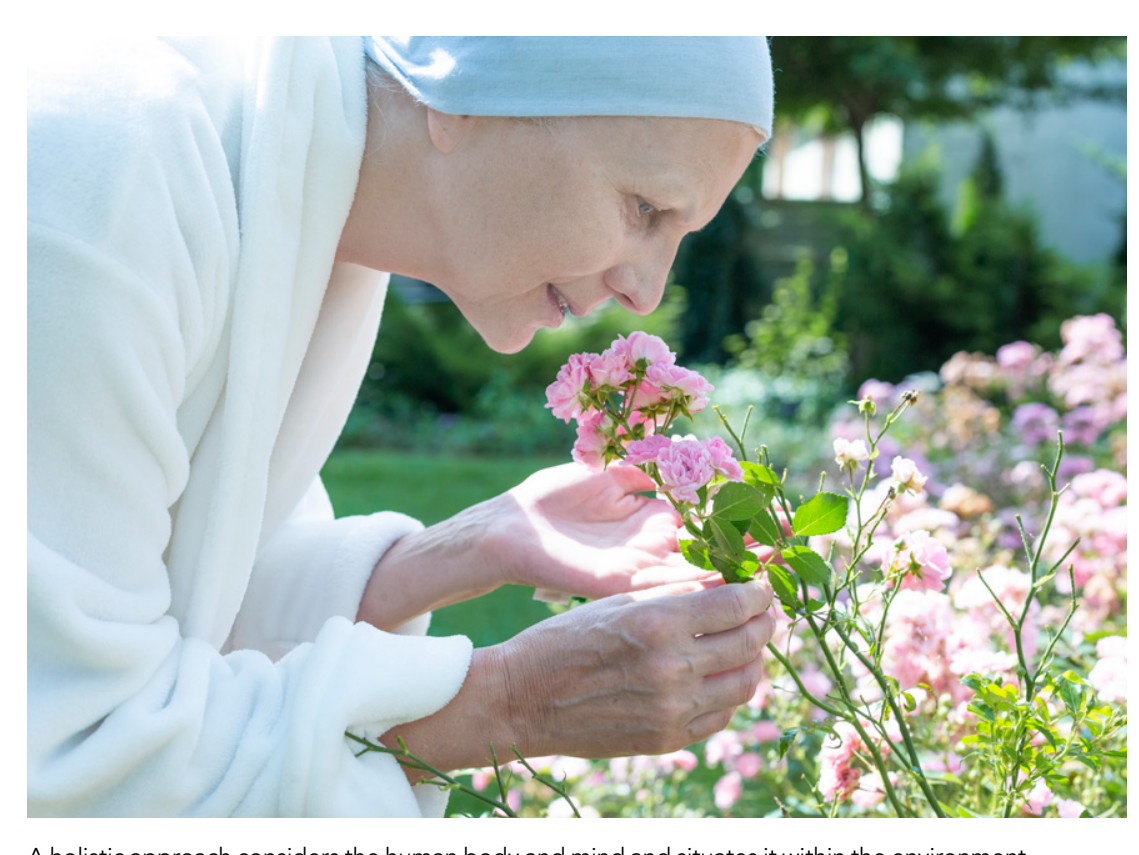

A holistic approach to medicine looks at a patient as an entire human being rather than a set of organs independently considered for the insurgence of a disease. thereby increasing the risk of disease. This concept is comparable to the Jungian pseudoego, characterised by overadaptation to the outside world whose role in development of cancer came to the attentic in the 1920 s.

ENVIRONMENTAL AND COLOGICAL FRAMEWORKS Cacies than in others. Risk factors commonly associated with cancer are also typically associated with industrialisation, such as smoking unhealthy diet, lack of physical activity, obesity, red meat intake, and certain infections. In particular, the Western diet, including processed foods, high intake of red meat, dairy, salt and sugar has been associated with increased risk of cancer across of genetics. Related studies have ently s. Related studies have centy shown that cancer is indeed need "a mory many factors, which determinants of resilence to the and endogenous challenge". This new approach considers the individual way
STRESS AS A MEDIATING FACTOR Although cancer has traditionally condition, recent research supports the hypothesis dating back to the early $20^{\text {th }}$ century that cancer, too, might be affected by emotions. While stress has been a muchdebated factor in cancer research, broader evidence suggests that stress alone does not result in cancer Nevertheless, stress does play a role in disease development by increasing an individual's vulnerability to infection, allergies, autoimmune conditions, and cardiovascular diseases, but there is not a direct link between str

\section{A HOLISTIC APPROACH}

A holistic approach to medicine looks at a patient as an entire human bein of physical parts, organs and systems considered as independent of each other. Organisms are open and active systems that organise, self-regulate, and self-differentiate. In people, this flux of information is conditioned upon the meaning we attribute to physiological and psychological experiences. These experiences, in turn, depend on the interaction between the cognitive and immunological systems in a single integrated system with a cybernetic organisation

Despite a growing body of evidence, today we can state that cancer has multifactorial origins but the interactive and psychological risk factors remal unclear. Future research needs to examine the human body and mind and their interaction with the environment. Given the complexity of the human organism, multidisciplinary research is required to bring these perspectives together for a more comprehensive understanding of the mechanisms of cancer. Consequently, not only prevention projects, but also therapeutic guidelines adding psychosocial support to the usual cancer treatments are necessary. A final proposal is, therefore, to provide an extensive cooperation between holstic and reductionist prevention and therapy.

\section{Behind the Research}

\section{Dr Marco Balenci}

A E: marco.balenci@gmail.com T: +39055 4379314

W: https://www.linkedin.com/in/marco-balenci-0799964b W: https://independent.academia.edu/MarcoBalenci

\section{Research Objectives}

Dr Balenci examines cancer holistically with an emphasis on looking at it as a systemic rather than local disease. Elida Evans on cancer (1926).

\section{Detail}

Marco Balenci

76 via Caduti di Cefalonia

50127 Florence

Italy

Bio

Marco Balenci, PhD, is a psychoanalyst, a member of the American Psychological Association, Associazion Italiana di Psicologia Analitica and International Association for Analytical Psychology. He worked at Sapienza University of Rome, co-edited five academic books, and published articles on health psychology and analytic concepts. He has a private practice in Florence.

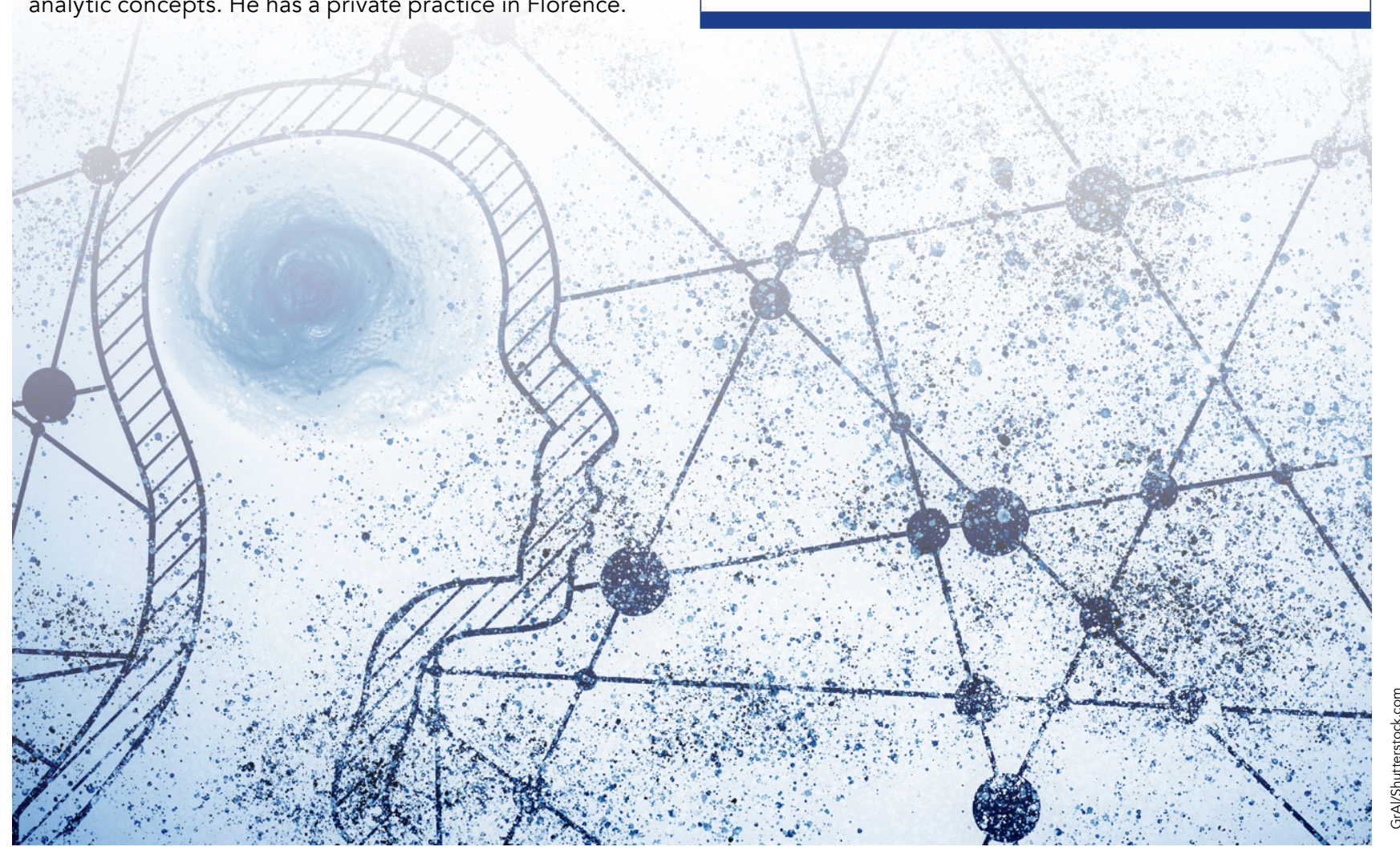

\section{References}

Balenci, M. (2019). Historical-clinical pathways to a cancer

\section{Personal Response}

In your opinion, what is the biggest obstacle to holistic medicine and psycho-oncology becoming

II Even today, psycho-oncology must confront the stigma of cancer and the mythology around it, as Susan Sontag origins outside of their biological aspects, particularly for their emotional implications. This concerns both patients and physicians, so that they prefer to focus on the sick the whole person with a holistic approach. It depends, not only on the reductionist conception of medicine bus ato on psychological motivations - which are very difficult to holistic perspective. Madridge J Cancer Stud Res, 3(1), 85-96. 